JAMA Insights / Clinical Update

Evidence-Based Approaches to Pain Management in 2019

\author{
Evan Wood, MD ${ }^{1}$ \\ David L. Simel, MD, $\mathrm{MHS}^{2}$ \\ Jan Klimas, $\mathrm{PhD}, \mathrm{MSc}^{1,3}$
}

1. Department of Medicine, University of British Columbia, Canada

2. Durham Veterans Affairs Medical Center and Duke University

Department of Medicine, Durham, NC 27705

3. School of Medicine, University College Dublin, Belfield, Dublin 4, Ireland

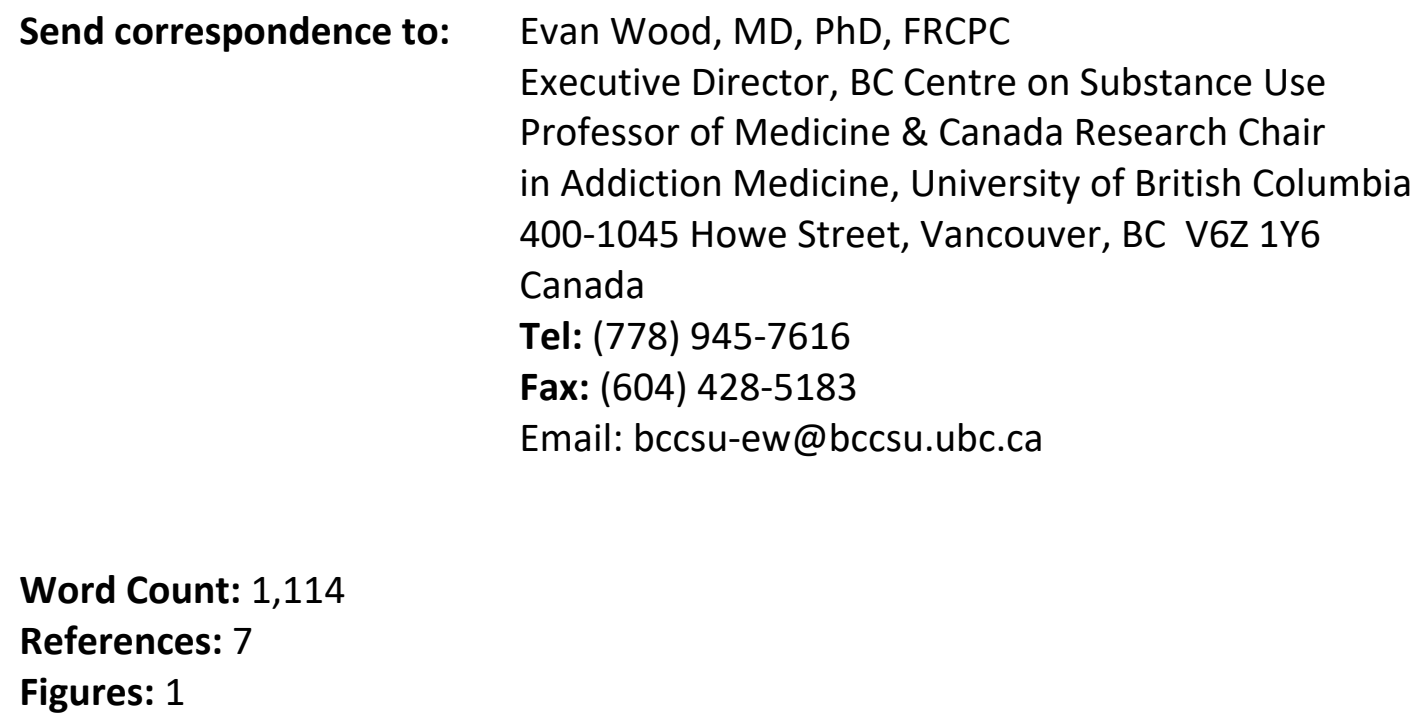

Figures: 1 
34 The evidence for the treatment of non-cancer pain among adults with acute and chronic

35 pain conditions has evolved rapidly in recent years. Earlier recommendations, which

36 have become increasingly acknowledged as being driven by misleading information

37 from opioid manufacturers, resulted in more routine use of opioid analgesics. As a

38 result, it has been estimated that almost $40 \%$ of U.S. civilian, non-institutionalized adults

39 used prescription opioids in $2015 .^{1}$ In this same study, almost $30 \%$ of those using

40 prescription opioids reported opioid misuse or opioid use disorder, and among those

41 with misuse, almost $60 \%$ reported using prescription opioids without a prescription. ${ }^{1}$

42 Coinciding with increased opioid prescribing has been a resurgence in illicit heroin use

43 and, more recently, the appearance of highly toxic illicitly manufactured fentanyl in the

44 street drug supply. Through these combined effects, it has been estimated that between

452016 and 2025, more than 700,000 individuals in the United States will die from opioid

46 overdose. $^{2}$

47 Beyond the health risks and harms associated with prescription opioid use,

48 recent research has clearly demonstrated that the promotion of more routine use of

49 prescription opioids was not evidence-based. For instance, a 2015 systematic review

50 concluded that the "evidence is insufficient to determine the effectiveness of long-term

51 opioid therapy" and instead concluded that "evidence supports a dose-dependent risk

52 for serious harms." ${ }^{3}$ A subsequent meta-analysis of 96 randomized controlled trials

53 involving more than 26,000 patients further demonstrated the limited clinical utility of

54 prescription opioids. ${ }^{4}$ Among patients receiving opioids for at least a month, benefits 
55 were insignificant in comparison to placebo (weighted mean difference, $-0.69 \mathrm{~cm}$

$56[95 \% \mathrm{Cl},-0.82$ to $-0.56 \mathrm{~cm}]$ on a $10-\mathrm{cm}$ visual analog scale for pain with a minimally

57 important difference of $1 \mathrm{~cm}$ ). Interestingly, while a wide range of oral opioid doses,

58 from $7.5 \mathrm{mg}$ to $242.7 \mathrm{mg}$ daily morphine equivalents, was considered, the analyses

59 demonstrated no difference in outcomes based on opioid dose. Importantly, since

60 modern pain care includes an array of alternatives to opioid-based analgesics, the most

61 clinically relevant part of this meta-analysis was the comparison of opioid and non-

62 opioid analgesics. Here, no differences in pain relief were observed between opioids and

63 opioid-sparing approaches. ${ }^{4}$

64 Since the evidence suggests that any acute benefit of opioid therapy on chronic

65 pain may diminish within weeks, ${ }^{4}$ a limitation of most research in this area is that most

66 opioid trials are very short (e.g., 6 weeks or less). ${ }^{3}$ To address this, the Strategies for

67 Prescribing Analgesics Comparative Effectiveness (SPACE) trial was a recent 12-month

68 pragmatic randomized trial of 240 patients that compared opioid analgesic therapy to

69 non-opioid medications for moderate to severe chronic back, hip or knee osteoarthritis

70 pain. ${ }^{5}$ Here, the trial demonstrated that treatment with opioids was not superior to

71 opioid alternatives for improving pain-related function. The authors concluded that the

72 results do not support the initiation of opioid therapy for moderate to severe

73 osteoarthritis pain. ${ }^{5}$

74 In response, recent pain guidelines, such as the U.S. Centers for Disease Control

75 and Prevention opioid prescribing guideline, highlight the importance of carefully

76 screening patients to identify those that are at high risk of opioid use disorder. Further, 
77 a host of screening instruments have been developed with a view to identifying patients

78 among whom opioid analgesics can safely be prescribed. However, until recently, these

79 recommendations and screening instruments have not been scrutinized for diagnostic

80 accuracy. To address this, we recently conducted a rigorous quality assessment and

81 critical appraisal of studies examining risk factors and risk screening instruments and

82 calculated sensitivity, specificity and likelihood ratios to assess the performance of

83 different measures. ${ }^{6}$ While the review indicated that a history of substance use disorder,

84 opioid prescriptions $\geqq 30$ days (and peak doses $>120$ milligrams morphine equivalents

85 per day), certain mental health diagnoses and prescription of certain concomitant

86 psychiatric medications appeared useful for identifying higher-risk patients, no

87 symptoms, signs or screening tools appeared useful for identifying those at lower risk.

88 Additionally, the review demonstrated that commonly used screening instruments, such

89 as the Opioid Risk Tool, provide no diagnostic value for clinicians. Collectively, while the

90 meaningful positive likelihood ratios for certain risk factors, such as having a history of

91 substance use disorder, suggest employing caution when prescribing opioid-based

92 medications in those with these risk factors, the absence of any identified useful

93 negative likelihood ratios suggests that the absence of identified risk factors does not

94 imply opioids can be safely prescribed. ${ }^{6}$

95 These findings coincide with increasing concerns that new prescribing guidelines

96 have resulted in harms from the withholding of opioid medications to those already on

97 opioid therapy for chronic pain. ${ }^{2}$ Most importantly, since in some cases these patients 
98 may turn to a street heroin market increasingly contaminated by toxic fentanyl analogs,

99 great caution must be employed in this context as well.

$100 \quad$ Based on recent literature and the rapidly evolving nature of the opioid overdose

101 epidemic due to the emergence of fentanyl analogs in the illicit drug supply, there are

102 clearly three main clinical scenarios being confronted by clinicians where evidence-

103 based recommendations can be made (Figure). The first clinical scenario is the approach

104 to consider for chronic pain patients (excluding cancer, palliative or other special

105 circumstances) not on opioid therapy. Here, the literature suggests that opioid therapy

106 should be avoided given the limited likelihood of benefit and the major evidence of

107 opioid-related harms, ${ }^{3}$ and that efforts to use the clinical examination or screening tools

108 to identify low-risk patients are likely of little value. ${ }^{6}$ The second clinical scenario is the

109 approach to consider with chronic pain patients already on opioid therapy. Here, an

110 approach involving individualized care is clearly warranted. ${ }^{2}$ Specifically, while the

111 literature suggests potential for improved pain and functioning with opioid tapering, this

112 must be balanced with the risks of exacerbating pain, opioid withdrawal syndrome and

113 the fact that withholding opioid therapies can potentially result in transition to street

114 opioid use. ${ }^{2}$ At the same time, given the prevalence and risks associated with

115 prescription opioid diversion and misuse, ${ }^{1}$ the proven benefits of opioid agonist therapy

116 in the prescription opioid addiction context, as well as the evidence that

117 buprenorphine/naloxone may provide similar analgesia as full opioid agonists, ${ }^{7}$ opioid

118 agonist therapy should be increasingly considered in this context. This will require that

119 efforts to overcome barriers to opioid agonist therapy be redoubled. ${ }^{2}$ The third clinical 
120 scenario is the approach to consider in acute pain contexts. Here, given that most

121 chronic pain initially presents as acute pain and the benefits of opioids on acute pain

122 may diminish rather quickly, ${ }^{4}$ and given the known risks of prolonged opioid prescription

123 and dose on risk of subsequent opioid addiction, ${ }^{6}$ opioid therapy should be avoided in

124 those with minor to moderate acute pain, and when opioids are used in severe acute

125 pain, the dose and duration limited to short (e.g., < 1 week), renewable (if necessary)

126 courses. $^{3}$

127 Acknowledgments

128 This research was undertaken, in part, thanks to funding from the Canada Research 129 Chairs program through a Tier 1 Canada Research Chair in Addiction Medicine, which 130 supports Dr. Evan Wood. A European Commission grant (701698) supports Dr. Klimas. 131 Dr. Simel receives honoraria for contributions to JAMAEvidence.com. Dr. Simel's work is 132 supported by the Durham Center of Innovation to Accelerate Discovery and Practice 133 Transformation (ADAPT, CIN 13-410) at the Durham VA Health Care System. We thank 134 Dr. Rashmi Chadha for her critical review of an earlier version of this manuscript and 135 Kevin Hollet and Deborah Graham for assistance with editing. 
Figure. Evidence-based opioid sparing pain management strategy.

\begin{tabular}{|c|c|c|}
\hline \multicolumn{3}{|c|}{ Acute and Chronic Pain } \\
\hline 1. Chronic Pain Off Opioids & 2. Chronic Pain on Opioids & 3. Acute Pain \\
\hline \multirow{3}{*}{$\begin{array}{l}\text { Avoid opioid therapy } \\
\text { Risk stratification not useful }\end{array}$} & Individualize treatment plan & \multirow{3}{*}{$\begin{array}{l}\text { Avoid opioids in minor to } \\
\text { moderate pain conditions } \\
\text { Limit opioid duration and } \\
\text { dose }^{b}\end{array}$} \\
\hline & Consider OAT if evidence & \\
\hline & of OUD & \\
\hline
\end{tabular}

Abbreviations: OAT, Opioid Agonist Therapy: OUD, Opioid Use Disorder.

"Buprenorphine/naloxone or methadone should be considered for patients with opioid use disorder -Opioid medications after major surgery or serious injury can be limited to short renewable courses

137 
140 1. Han B, Compton WM, Blanco C, Crane E, Lee J, Jones CM. Prescription opioid use, 141 misuse, and use disorders in U.S. adults: 2015 National Survey on Drug Use and 142 Health. Annals of internal medicine. 2017;167(5):293-301.

143 2. Chen Q, Larochelle MR, Weaver DT, et al. Prevention of prescription opioid 144 misuse and projected overdose deaths in the United States. JAMA network open. 145 2019;2(2):e187621.

146 3. Chou R, Turner JA, Devine EB, et al. The effectiveness and risks of long-term opioid therapy for chronic pain: a systematic review for a National Institutes of Health Pathways to Prevention Workshop. Annals of internal medicine. 2015;162(4):276-286.

4. Busse JW, Wang L, Kamaleldin M, et al. Opioids for chronic noncancer pain: a systematic review and meta-analysis. JAMA. 2018;320(23):2448-2460.

5. Krebs EE, Gravely A, Nugent S, et al. Effect of opioid vs nonopioid medications on pain-related function in patients with chronic back pain or hip or knee osteoarthritis pain: the SPACE randomized clinical trial. JAMA. 2018;319(9):872882.

6. Klimas J, L. G, Fairbairn N, et al. Strategies to identify patient risks of prescription opioid addiction when initiating opioids for pain: a systematic review. JAMA network open (in press). 2019.

7. Raffa RB, Haidery M, Huang HM, et al. The clinical analgesic efficacy of buprenorphine. Journal of clinical pharmacy and therapeutics. 2014;39(6):577583. 
Figure. Evidence-based opioid sparing pain management strategy.

\section{Acute and Chronic Pain}

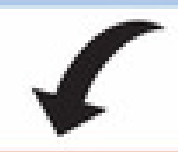

1. Chronic Pain Off Opioids

Avoid opioid therapy ${ }^{3,4}$

Risk stratification not useful ${ }^{6}$

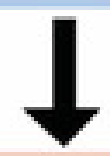

2. Chronic Pain on Opioids

Individualize treatment plan

Consider OAT if evidence

of OUD

\section{Acute Pain}

Avoid opioids in minor to moderate pain conditions

Limit opioid duration and dose $^{b}$

Abbreviations: OAT, Opioid Agonist Therapy; OUD, Opioid Use Disorder.

a Buprenorphine/naloxone or methadone should be considered for patients with opioid use disorder

- Opioid medications after major surgery or serious injury can be limited to short renewable courses 